Check for updates

London, UK

Cite this as: BMJ 2021;373:n954 http://dx.doi.org/10.1136/bmi.n954 Published: 12 April 2021

\section{Covid-19: Rare immune response may cause clots after AstraZeneca vaccine, say researchers}

\author{
Jacqui Wise
}

Two teams of researchers have published detailed observations of patients who developed thrombotic thrombocytopenia after receiving the AstraZeneca vaccine and have speculated about a possible mechanism. ${ }^{12}$

Both groups suggest that the development of serious blood clots alongside falling levels of platelets is an immune response that resembles a rare reaction to the drug heparin, called heparin induced thrombocytopenia. The researchers have labelled the syndrome vaccine induced immune thrombotic thrombocytopenia.

Among more than 20 million people who have been vaccinated with the AstraZeneca vaccine in the UK so far, 79 cases of rare blood clots with low platelets have been reported, as well as 19 deaths. The European Medicines Agency and the UK's Medicines and Healthcare Regulatory Agency have concluded that unusual blood clots with low blood platelets are a possible and rare side effect of the AstraZeneca vaccine but a causal relation has not been established. ${ }^{3}$ The EMA's Pharmacovigilance Risk Assessment Committee is also investigating three cases of unusual blood clots with low blood platelets during the vaccine rollout of the Johnson \&Johnson Janssen vaccine in the US.

The first paper, published in the New England Journal of Medicine, describes 11 patients in Austria and Germany, 9 of them women, with a median age of 36 years. They had clotting and low platelets between five to 16 days after vaccination. Nine patients had cerebral venous thrombosis, three had splanchnic vein thrombosis, three had pulmonary embolism, and four had other thromboses. Six of the patients died.

All 11 patients, as well as another 17 for whom the researchers had blood samples, tested positive for antibodies against platelet factor 4 ( $P F 4)$. These antibodies are also observed in people who develop heparin induced thrombocytopenia. None of the patients had received heparin before their symptoms started, however.

"Whether these antibodies are autoantibodies against PF4 induced by the strong inflammatory stimulus of vaccination or antibodies induced by the vaccine that cross-react with $\mathrm{PF}_{4}$ and platelets requires further study," wrote Andreas Greinacher from the University of Griefswald in Germany, and his co-authors. They suggested that one possible trigger of these $\mathrm{PF}_{4}$ reactive antibodies could be free DNA in the vaccine.

In a second paper, also published in the New England Journal of Medicine, researchers in Norway described a similar pattern in five healthcare workers who were 32 to 54 years old. All had high levels of PF4 antibodies and no previous exposure to heparin. Four of the patients had major cerebral haemorrhage and three died.

Both sets of researchers suggested that doctors should have a low threshold for requesting enzyme linked immunosorbent assay testing for $\mathrm{PF}_{4}$-heparin antibodies in patients who have unexpected symptoms after vaccination. They suggested treatment with intravenous immune globulin and non-heparin blood thinners.

Commenting on the studies, Peter English, retired consultant in communicable disease control, former editor of Vaccines in Practice, and immediate past chair of the BMA Public Health Medicine Committee, said, "These papers do not tackle causality. They may turn out to be useful, in their technical details, for clinicians treating patients with clotting disorders, and for regulators and others in deciding the safest way to proceed once causality has been confirmed-if it ever is. However, their small studies do not have the statistical power to, and are not designed to, prove causality"

On 7 April, the UK's Expert Haematology Panel published guidance for the diagnosis and management of vaccine induced thrombosis and thrombocytopenia which they say is rare but can affect patients of all ages and both sexes. ${ }^{4}$ They recommended urgent use of intravenous immunoglobulin, avoiding platelet transfusions, and anticoagulating with non-heparin based therapies. Probable cases must be reported to the panel and Public Health England at https://cutt.ly/haem_AE. Additionally, all cases of thrombosis or thrombocytopenia occurring within 28 days of covid-19 vaccine must be reported to the Medicines and Healthcare Products Regulatory Agency through the online yellow card system (https://coronavirusyellowcard.mhra.gov.uk).

The MHRA has recommended that anyone who develops the following symptoms after vaccination should seek prompt medical advice-shortness of breath, chest or persistent abdominal pain, leg swelling, blurred vision, confusion of seizures, unexplained pin prick rash, or bruising beyond the injection site.

Greinacher A, Thiele T, Warkentin TE, Weisser K, Kyrle PA, Eichinger S. Thrombotic thrombocytopenia after ChAdOx1 nCov-19 vaccination. N Engl J Med 2021. doi: 10.1056/NEJMoa2104840. pmid: 33835769

2 Schultz NH, Sørvoll IH, Michelsen AE, et al. Thrombosis and thrombocytopenia after ChAdOx1 nCoV-19 vaccination. N Engl J Med2021. doi: 10.1056/NEJMoa2104882. pmid: 33835768

3 Mahase E. AstraZeneca vaccine: Blood clots are "extremely rare" and benefits outweigh risks, regulators conclude. BMJ2021;373:n931. doi: 10.1136/bmj.n931 pmid: 33832929 
4 Guidance produced from the Expert Haematology Panel focused on covid-19 vaccine induced thrombosis and thrombocytopenia. 7 April 2021. https://b-s-h.org.uk/media/19530/guidanceversion-13-on-mngmt-of-thrombosis-with-thrombocytopenia-occurring-after-c-19-vac-

$$
\text { cine_20210407.pdf. }
$$

This article is made freely available for use in accordance with BMJ's website terms and conditions for the duration of the covid-19 pandemic or until otherwise determined by BMJ. You may use, download and print the article for any lawful, non-commercial purpose (including text and data mining) provided that all copyright notices and trade marks are retained. 